\title{
Application of Scale Relativity to the Problem of a Particle in a Simple Harmonic Oscillator Potential
}

\author{
Saeed N. T. Al-Rashid', Mohammed A. Z. Habeeb' ${ }^{2}$, Khalid A. Ahmed ${ }^{3}$ \\ ${ }^{1}$ Physics Department, College of Pure Science, University of Anbar, Anbar, Iraq \\ ${ }^{2}$ Physics Department, College of Science, Al-Nahrain University, Baghdad, Iraq \\ ${ }^{3}$ Physics Department, College of Science, Al-Mustansiriyah University, Baghdad, Iraq \\ Email: sntr2006@yahoo.com
}

How to cite this paper: Al-Rashid, S.N.T., Habeeb, M.A.Z. and Ahmed, K.A. (2017) Application of Scale Relativity to the Problem of a Particle in a Simple Harmonic Oscillator Potential. Journal of Quantum Information Science, 7, 77-88. https://doi.org/10.4236/jqis.2017.73008

Received: July 22, 2017

Accepted: September 11, 2017

Published: September 18, 2017

Copyright $\odot 2017$ by authors and Scientific Research Publishing Inc. This work is licensed under the Creative Commons Attribution International License (CC BY 4.0).

http://creativecommons.org/licenses/by/4.0/

\begin{abstract}
In the present work, Scale Relativity (SR) is applied to a particle in a simple harmonic oscillator (SHO) potential. This is done by utilizing a novel mathematical connection between SR approach to quantum mechanics and the well-known Riccati equation. Then, computer programs were written using the standard MATLAB 7 code to numerically simulate the behavior of the quantum particle utilizing the solutions of the fractal equations of motion obtained from SR method. Comparison of the results with the conventional quantum mechanics probability density is shown to be in very precise agreement. This agreement was improved further for some cases by utilizing the idea of thermalization of the initial particle state and by optimizing the parameters used in the numerical simulations such as the time step and number of coordinate divisions. It is concluded from the present work that SR method can be used as a basis for description the quantum behavior without reference to conventional formulation of quantum mechanics. Hence, it can also be concluded that the fractal nature of space-time implied by SR, is at the origin of the quantum behavior observed in these problems. The novel mathematical connection between SR and the Riccati equation, which was previously used in quantum mechanics without reference to $S R$, needs further investigation in future work.
\end{abstract}

\section{Keywords}

Simple Harmonic Oscillator, Scale Relativity, Numerical Simulations, Fractal Space-Time 


\section{Introduction}

Scale relativity (SR) developed by Nottale based on the extension of the principle relativity as follows "the fundamental laws of nature apply whatever the state of scale of the coordinate system" [1] [2] [3] [4] [5]. The observation resolutions now characterize the reference system and can be defined only in relative way. This major concept of SR leads to giving up hypothesis of differentiability of space-time. Quantum mechanics can then be reformulated from this basic principle of SR form of covariance and geodesic equations, by considering a particle as a geodesics in now fractal space-time. There are at least three major fields of application for SR method, microphysics, complex systems and cosmology [6] [7] [8] [9] [10].

As far as quantum mechanics is concerned, Nottale and co-workers were able to apply the theory to solve many problems, especially those related to the conceptual and interpretation aspects. The derivation of the postulates of quantum mechanics from basic principle of SR [11], is basis of the present work. It shows that quantum mechanical behavior appears without any use of the Schrodinger equation, but as a consequence of the fractality of space-time. The extension of the SR theory to the derivation of the main equations of relativistic quantum mechanics [12] and the relationship between the classical and quantum regimes [13] have been also discussed on the basis of the SR among other important consequences and implications. With all these far reaching aspects of the theory, direct investigations which would shed light on the basic workings of the SR method as formulated by Nottale seem to be warranted.

The fractional equations of motion which are obtained from application of SR, were applied directly by Hermann [14], in terms of a large number of explicit numerically simulated trajectories for a free particle in an infinite one-dimensional box [15] [16] [17]. Similarly, Al-Rashid [18] [19] [20], applied SR to the finite one-dimensional square well potential and special case in a double oscillator problems.

The validity of SR not restricted to the cases by Hermann [14] and Alrashid [18] [19] [20]. Besides, such applications are expected to reveal some novel concepts, such as the connection between SR and the Riccati equation [21] [22] [23] as revealed in the present work.

In this paper, the problem of a particle moving in one dimensional SHO will be treated by applying the principle of SR along the lines of Hermann. To the best of our knowledge, this problem has not been treated by using Hermann line elsewhere [14] [24].

\section{Equation of Motion}

One may start with the complex Newton Equation [14] [18]:

$$
\nabla u=m \frac{\partial}{\mathrm{d} t} \mathbf{V}
$$

where $u$ is a scalar potential and $\mathrm{V}$ is a complex velocity, then separate this equa- 
tion into real and imaginary parts:

$$
\begin{aligned}
& m\left(\frac{\partial}{\partial t} V-D \Delta U+(V \cdot \nabla) V-(U \cdot \nabla) U\right)=-\nabla u \\
& \text { and } m\left(\frac{\partial}{\partial t} V+D \Delta V+(V \cdot \nabla) U+(U \cdot \nabla) V\right)=0
\end{aligned}
$$

Here, the average classical velocity $V$ is expected to be zero because the simple harmonic oscillator is a symmetric system. Then, the equations of motion can be reduced as [14] [18]:

$$
\frac{\partial}{\partial x}\left(\frac{\partial}{\partial x} D U(x)+\frac{1}{2} U^{2}(x)\right)=0
$$

and

$$
\frac{\partial}{\partial t} U(x)=0
$$

where $U$ is the imaginary part of complex velocity and $D$ is the diffusion coefficient. Equation (4) shows that $U$ is a function of $x$ alone. The potential of the one-dimensional SHO can be written as $\frac{1}{2} m \omega^{2} x^{2}$, where $\omega$ is the angular frequency. Then, Equation (3) becomes:

$$
\frac{\partial}{\partial x}\left(\frac{\partial}{\partial x} D U(x)+\frac{1}{2} U^{2}(x)\right)=\frac{1}{2} m \omega^{2} \frac{\partial}{\partial x} x^{2}
$$

Integrating and rearranging terms in the resulting equation, one obtains:

$$
\frac{\mathrm{d}}{\mathrm{d} x} U(x)+\frac{1}{2 D} U^{2}(x)-\frac{1}{2 D} m \omega^{2} x^{2}+\frac{1}{D} c_{1}=0
$$

where $c_{1}$ is a constant of integration. Letting $c_{1}=E / m$ (as in Hermann's work) [14], then Equation (6) becomes:

$$
\frac{\mathrm{d} U(x)}{\mathrm{d} x}+\frac{m}{\hbar} U^{2}(x)-\frac{m^{2}}{\hbar} \omega^{2} x^{2}+\frac{2 E}{\hbar}=0
$$

where $D=\frac{\hbar}{2 m}$. The last equation has the form of a Riccati equation [19] [20] [21] [22] [23]. To solve this equation, one may trans-form it into a $2^{\text {nd }}$ order differential equation of the form [19] [20] [21] [22] [23]:

$$
r y^{\prime \prime}(x)+r^{2} q(x) y(x)=0
$$

where,

$$
U(x)=-\frac{1}{r} \frac{y^{\prime}(x)}{y(x)}
$$

and $y(x)$ is an arbitrary function of $x$. From this Equation (7), it follow that:

$$
r=-\frac{m}{\hbar} ; q(x)=\frac{2}{\hbar}\left(\frac{1}{2} m \omega^{2} x^{2}-E\right)
$$

Then, Equation (7) becomes: 


$$
y^{\prime \prime}(x)+\frac{2 m}{\hbar^{2}}\left(E-\frac{1}{2} m \omega^{2} x^{2}\right) y(x)=0
$$

Its solution is:

$$
y_{n}(x)=A_{n} \exp \left(-\frac{x^{2}}{2}\right) H_{n}(x)
$$

where $A_{n}$ is a constant and $H_{n}$ is a Hermite polynomial of order $n$ and $n=0,1,2, \cdots$. Then, $U_{n}(x)$ is given by:

$$
U_{n}(x)=\frac{\hbar}{m}\left(-x H_{n}(x)+H_{n}^{\prime}(x)\right)
$$

Using the equality $H_{n}^{\prime}(x)=x n H_{n-1}(x)$ then, Equation (13) becomes:

$$
U_{n}(x)=\frac{\hbar}{m}\left(-x+2 n\left(H_{n-1}(x) / H_{n}(x)\right)\right)
$$

As in Hermann work [14], $U(x)$ is treated as a difference of velocities, i.e., it is a kind of acceleration. Thus, the equation of position coordinate has the following form, which is a stochastic process:

$$
\mathrm{d} x(t)=\frac{\hbar}{m}\left(-x+2 n\left(H_{n-1}(x) / H_{n}(x)\right)\right) \mathrm{d} t+\mathrm{d} \xi_{+}(t)
$$

where $\mathrm{d} \xi_{+}(t)$ is now Gaussian random variable of standard deviation $\sqrt{2 D \mathrm{~d} t}$

\section{Numerical Simulations}

Equation (15) represents a stochastic process [14]. Here, in the problem of a one-dimensional SHO, it was found that the assumption $2 D \mathrm{~d} t=1$ is not useful for the present simulations since it gives bad results for the present application. Then, one starts to adjust the value of $\mathrm{dt}$ until one approaches a specific value for which meaningful results are obtained. It was found that a value of $\mathrm{d} t=10^{-3} \frac{\mathrm{m}}{\hbar}$ is suitable for the present simulations. It seems that this value of $\mathrm{dt}$ is related to the period of the motion in the SHO potential. It is expected that a suitable value which gives meaningful numerical simulation results is that which leads to a sufficient number of time steps during one period so as to give meaningful counts. This is a consequence of the statistical nature of these simulations which requires better statistics to be meaningful. Then, Equation (15) becomes:

$$
\mathrm{d} x(t)=10^{-3}\left(-x+2 n\left(H_{n-1}(x) / H_{n}(x)\right)\right)+\sqrt{10^{-3}} N(0,1)
$$

where the choice of units was made such that $\hbar=m=1$.

A computer program was written (see Appendix A), following Hermann's procedure [14], to make numerical simulations for the SHO problem. Numerical simulations are performed using Equation (16) which represent trajectory equations of the particle for different different values of the quantum number $n(n=$ $0,1,2,3,4$ and 5). The output of these simulations gives the probability density $f(x)$ of the particle in simple harmonic oscillator potential. To construct it, one 
may divide the region into 601 pieces (bins), which gives the best results and time steps (cc) of $10^{8}$ and $5 \times 10^{8}$ steps were used, as in Hermann's work.

The results of the present numerical simulations are compared with the probability density of conventional quantum mechanics, that is, $P(x)=N_{n}^{2} H_{n}^{2}(x) \mathrm{e}^{-x^{2}}$ where $N_{n}=1 / \sqrt{2^{n} n ! \pi^{1 / 2}}$ is the normalization constant [15] [16] [17]. The continuous curves indicate the results of the present simulations and the dashed curves the results of conventional quantum mechanics, with the same normalization as the numerical results. The comparison between the present results and the results of conventional quantum mechanics is further facilitated by calculating the standard deviation $\sigma$ and correlation coefficient $\rho$, which are given by [14]:

$$
\sigma=\sqrt{\frac{\sum_{i=1}^{N}(P(i)-f(i))^{2}}{N}}
$$

and

$$
\rho=\frac{\sum_{i=1}^{N}(P(i)-\langle P\rangle)(f(i)-\langle f\rangle)}{\sum_{i=1}^{N}(P(i)-\langle P\rangle)^{2} \sum_{i=1}^{N}(f(i)-\langle f\rangle)^{2}}
$$

where $N$ is the number of pieces, $P(i) \equiv P(x)$ and $f(i) \equiv f(x)$.

Figures 1-3 show the results of numerical simulations for $n=0,1,2,3,4$, and 5 with $10^{8}$ time steps (cc). These numerical simulations started with arbitrary particle at the position $x=2$. Also, the output of the simulations was normalized by multiplying it with a constant $q$ whose value depends on the number of divisions of the region (here, $q=50$ ).

Here, it was found, after some numerical tests, that the thermalization process [14] is useful to improve the present results. Figure 4 shows the results of such numerical tests for $n=2,3$ and 5 which have starting points ss $=100$ and 200 . These starting points are chosen after many attempts and were found to give better results from other choices. The improvement is clear from the values of $\sigma$

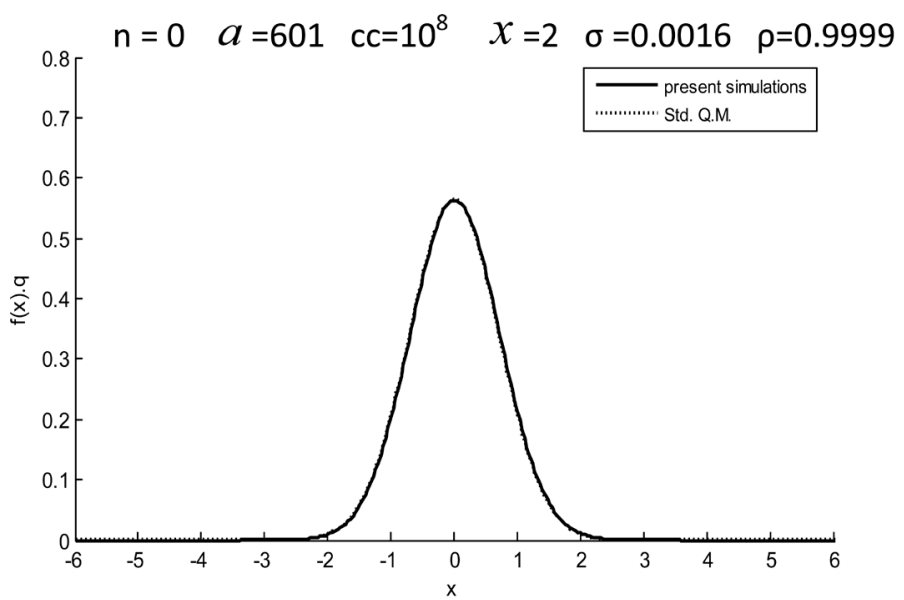

(a) 


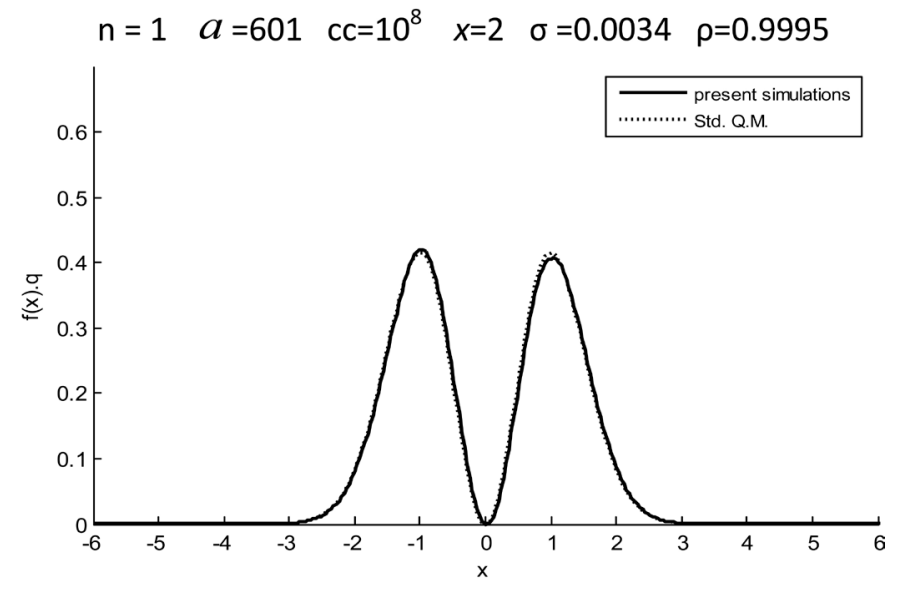

(b)

Figure 1. Probability density for a particle in a SHO potential (a) $n=0$ and (b) $n=1$, without thermalization process.

$\mathrm{n}=2 \quad a=601 \quad \mathrm{cc}=10^{8} \quad x=2 \quad \sigma=0.0127 \quad \rho=0.9914$

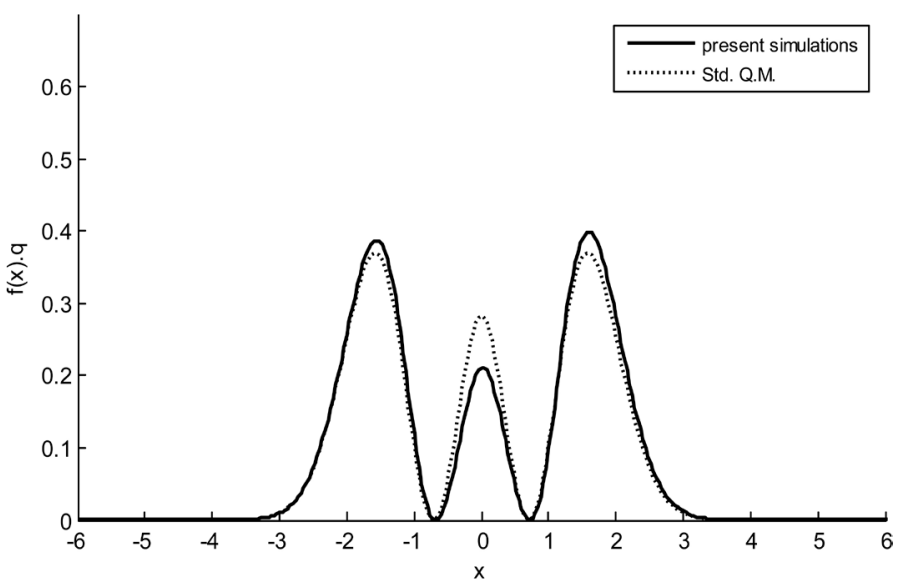

(a)

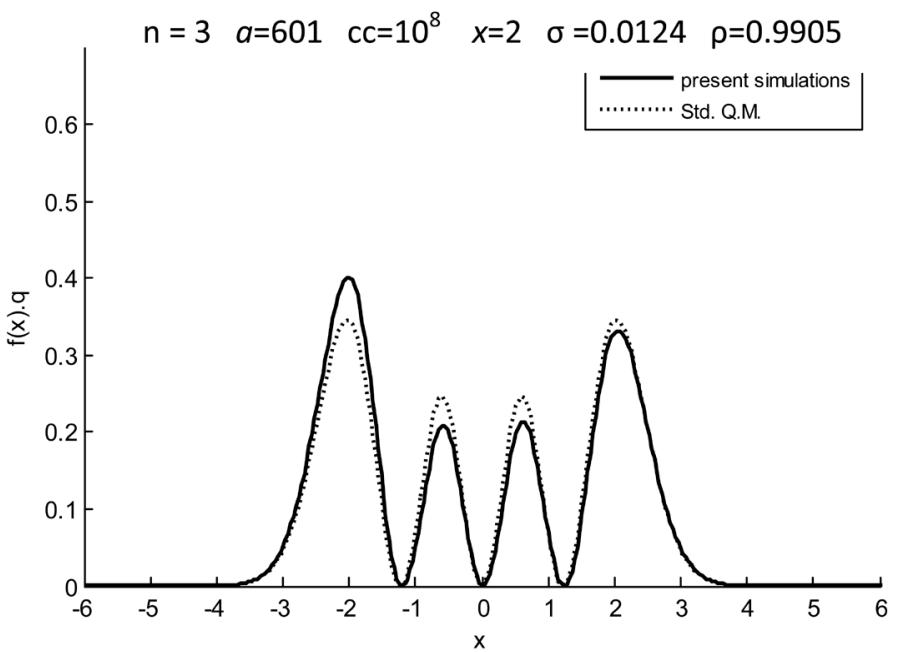

(b)

Figure 2. Probability density for a particle in a SHO potential (a) $n=2$ and (b) $n=3$, without thermalization process. 


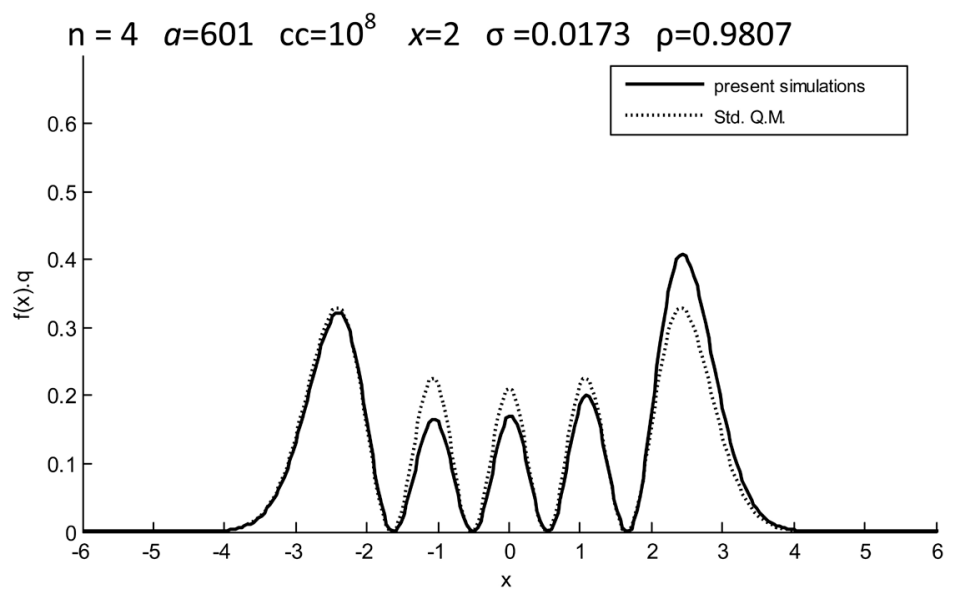

(a)

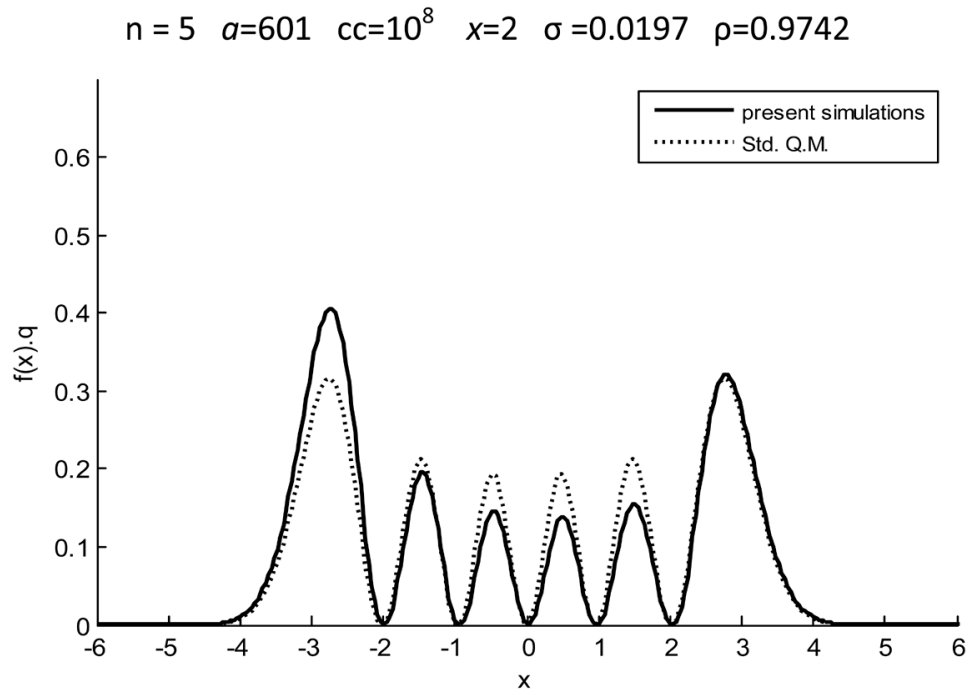

(b)

Figure 3. Probability density for a particle in a SHO potential (a) $\mathrm{n}=4$ and (b) $\mathrm{n}=5$, without thermalization process.

and $\rho$ compared with Figure 2 and Figure 3. The present results can also be improved to increase convergence between them and the results of quantum mechanics by using more time steps. Figure 5 shows the results obtained this way, for $n=3$. It appears that there is a better agreement with the results of conventional quantum mechanics compared with the results from a thermalization process for $n=3$ (see Figure 4 ).

It was also found that, in the present problem, convergence between the results of numerical simulations and those of conventional quantum mechanics can be improved by increasing the number of boxes. This is clear in Figure 6, where it appears that there is better agreement between the two results for $n=3$ when the number of boxes was increased to 1201 .

\section{Conclusion}

The quantitative prediction of the behavior of a quantum particle in simple 
harmonic oscillator potential can be correctly obtained without explicitly writing the Schrödinger equation nor using any other of the conventional quantum axiom. This leads one to conclude from the present work that SR is a wellfounded approach for deriving quantum mechanics from the concept of fractal space-time, consequence of the extension of the relativity principle to resolu-

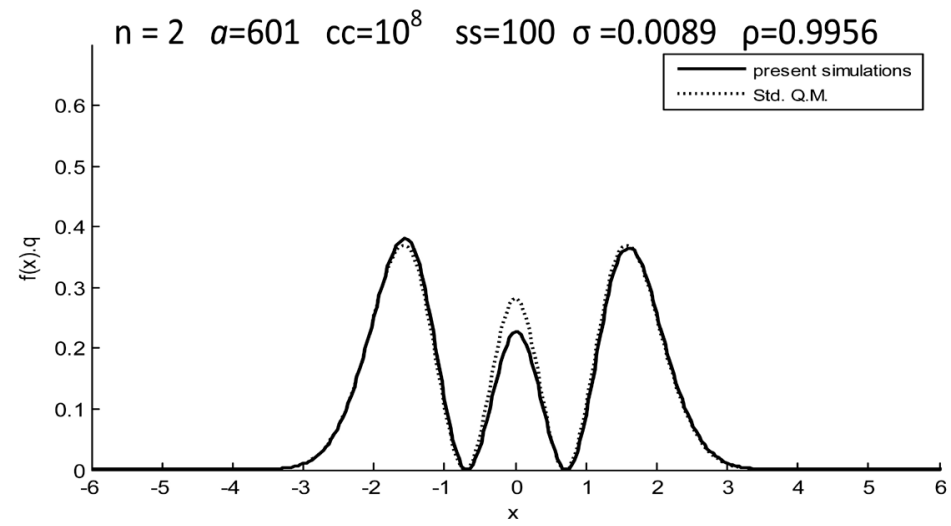

(a)

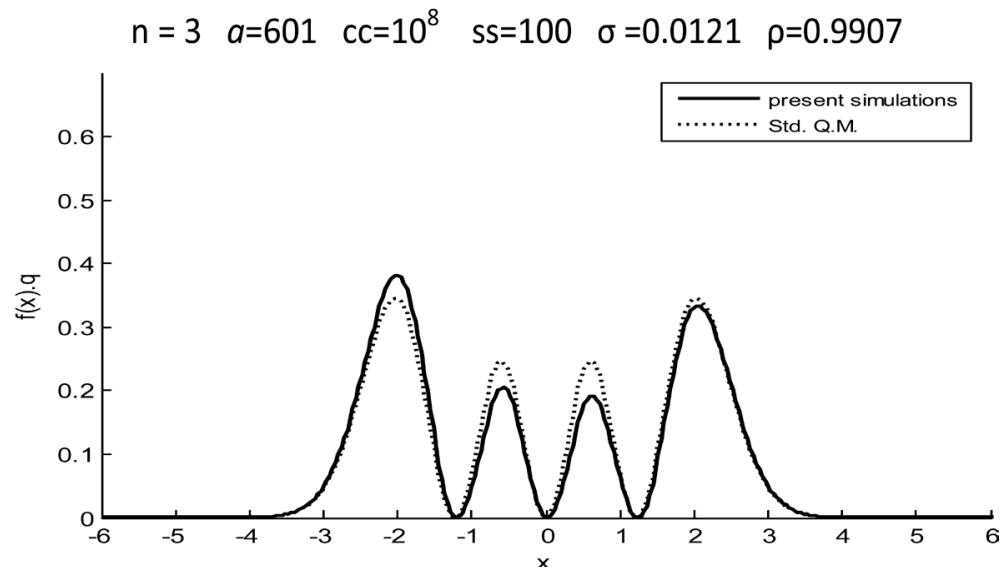

(b)

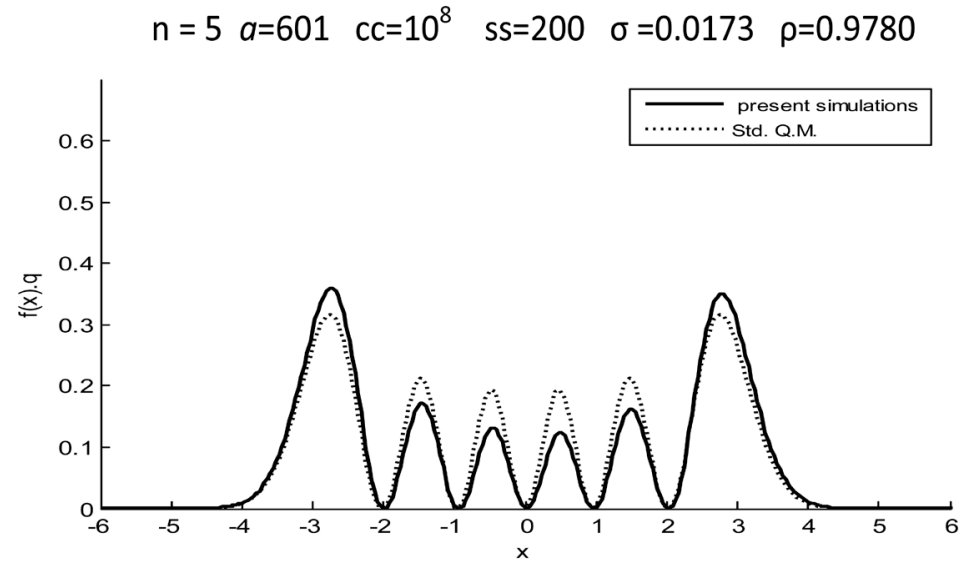

(c)

Figure 4. Probability density for a particle in a SHO potential (a) $n=2$, (b) $n=3$ and (c) $n=5$, with thermalization process. 


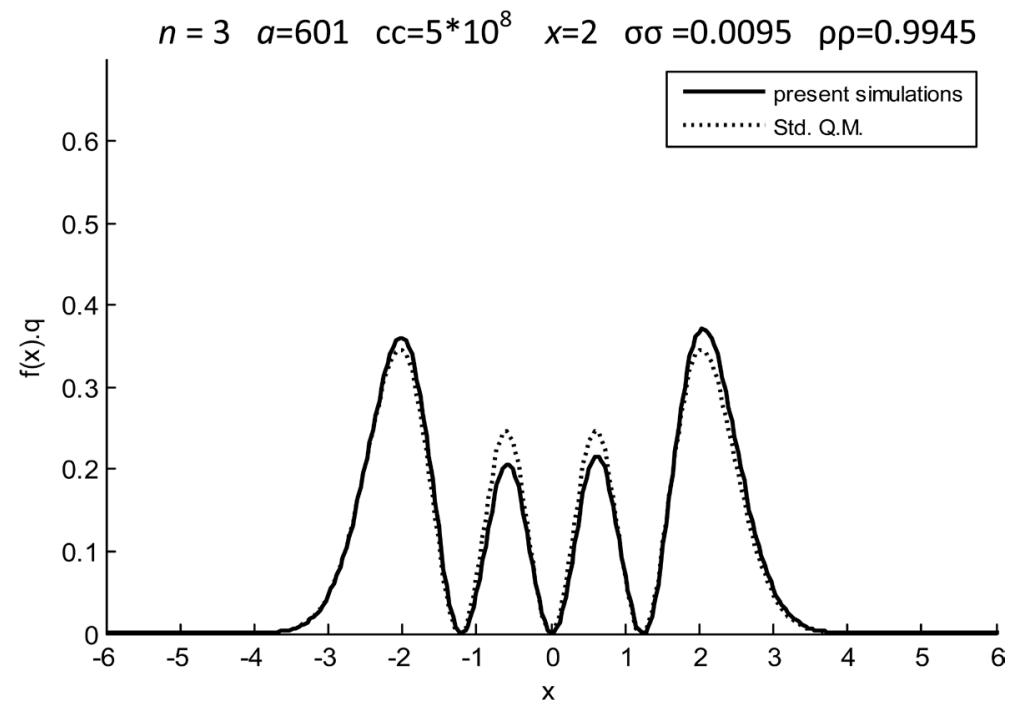

Figure 5. Probability density for a particle in a SHO potential with $n=3$ for longer time steps $\left(\mathrm{cc}=5 \times 10^{8}\right)$.

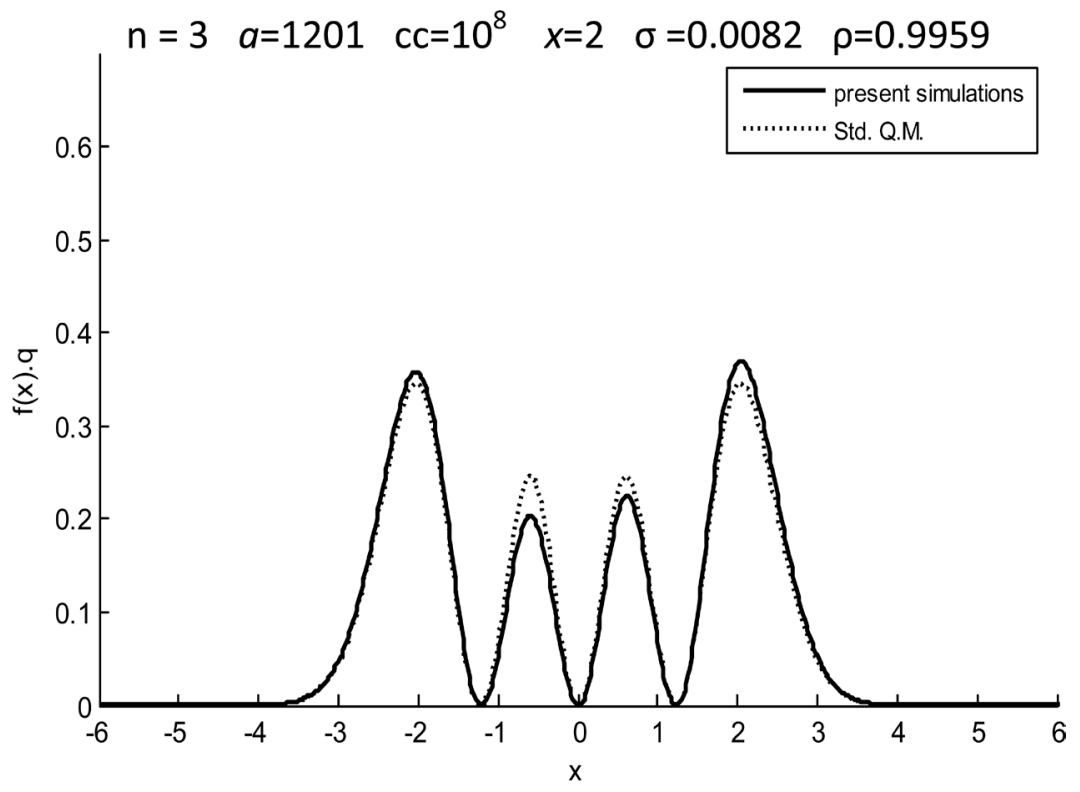

Figure 6. Probability density for a particle in a SHO potential with $\mathrm{n}=3$ after increasing the number of boxes.

tions. Successful applications were not achievable without, among other things, a new adjustment for the time step dt after some deeper understanding of the underlying particle motion in some problems. It is expected that this understanding is necessary when attempts are made to solve other quantum mechanical problems. The appearance of the Riccati equation in connection with SR theory in the present work, and the use of this equation in conventional quantum mechanics in previous works [22] [23] leads one to conclude that this equation is deeply rooted in the quantum mechanical behavior. It is also concluded from the attempts made in the present work that it is possible to improve the numerical simulation results by parameter optimization, and that further improvement is 
possible, but requires more computer time. SR is not a particularly advantageous approach for solving quantum mechanical problems directly. Rather, reveals the relationship between the quantum behavior and the fractality of space-time.

\section{Acknowledgements}

We would like to deeply thank Prof. Dr. L. Nottale (Director of Research, CNRS, Paris, France) for clarifying some points regarding his theory of scale relativity, Dr. R. Hermann (Dept. of Physics, Univ. de Liege, Belgium) for his suggestions concerning further applications of scale relativity method and Dr. Stephan LeBohec (Dept. Physics and Astronomy, Univ. of Utah, Salt Lake City, Utah, USA) for his continuous encouragement.

\section{References}

[1] Nottale, L. (1998) Fractal Space-Time and Microphysics: To wards of a Theory of Scale Relativity. World Scientific (First Reprint).

[2] Nottale, L. (1994) The Scale Relativity Program. Chaos, Solitons and Fractals, 10, 459-468. https://doi.org/10.1016/S0960-0779(98)00195-7

[3] Nottale, L. (1992) The Theory of Scale Relativity. International Journal of Modern Physics A, 7, 4899-4936. https://doi.org/10.1142/S0217751X92002222

[4] Nottale, L. (2004) The Theory of Scale Relativity: Non-Differentiable Geometry and Fractal Space-Time. AIP Conference Proceedings, 718, 68-95. https://doi.org/10.1063/1.1787313

[5] Nottale, L. (1996) Scale Relativity and Fractal Space-Time: Application to Quantum Physics, Cosmology and Chaotic Systems. Chaos, Solitons and Fractals, 7, 877-938. https://doi.org/10.1016/0960-0779(96)00002-1

[6] Nottale, L. (1997) Scale Relativity and Quantization of the Universe-I, Theoretical Framework. Astronomy \& Astrophysics, 327, 867-889.

[7] Nottale, L., Schumacher, G. and Gray, J. (1997) Scale Relativity and Quantization of the Solar System. Astronomy \& Astrophysics, 322, 1018-1025.

[8] Nottale, L. (1996) Scale Relativity and Quantization of Extra-Solar Planetary Systems. Astronomy \& Astrophysics, 315, L9-L12.

[9] Nottale, L. (1998) Scale Relativity and Quantization of the Planetary Systems around the Pulsar PSR B1257+12. Chaos, Solitons and Fractals, 9, 1043-1050. https://doi.org/10.1016/S0960-0779(97)00079-9

[10] Nottale, L. (1998) Scale Relativity and Quantization of Planet Obliquities. Chaos, Solitons and Fractals, 9, 1035-1041. https://doi.org/10.1016/S0960-0779(97)00078-7

[11] Nottale, L. and Célérier, M.N. (2007) Derivation of the Postulates of Quantum Mechanics from the First Principles of Scale Relativity. Journal of Physics A: Mathematical and Theoretical, 40, 14471-14498.

https://doi.org/10.1088/1751-8113/40/48/012

[12] Célérier, M.N. and Nottale, L. (2010) Electromagnetic Klein-Gordon and Dirac Equations in Scale Relativity. International Journal of Modern Physics A, 25, 4239-4253. https://doi.org/10.1142/S0217751X10050615

[13] Nottale, L. (2005) On the Transition from the Classical to the Quantum Regime in Fractal Space-Time Theory. Chaos, Solitons and Fractals, 25, 797-803.

[14] Hermann, R.P. (1997) Numerical Simulation of a Quantum Patrical in a Box. Jour- 
nal of Physics A: Mathematical and General, 30, 3967-3975. https://doi.org/10.1088/0305-4470/30/11/023

[15] Schiff, L.I. (1969) Quantum Mechanics. 3rd Edition, Int. Student, McGraw-Hill.

[16] Gasiorowicz, S. (1974) Quantum Physics. John Wiley and Sons, Inc., New York.

[17] Powell, J.L. and Crasemann, B. (1961) Quantum Mechanics. Addison-Wesley Publishing Co., Inc.

[18] Alrashid, S.N.T., Habeeb, M.Z.A. and Ahmed, K.A. (2011) Application of Scale Relativity (ScR) Theory to the Problem of a Particle in a Finite One-Dimensional Square Well (FODSW) Potential. Journal of Quantum Information Science, 1, 7-17. https://doi.org/10.4236/jqis.2011.11002

[19] Al-Rashid, S.N.T. (2006) Some Applications of Scale Relativity Theory in Quantum Physics. PhD Thesis, Al-Mustansiriyah University.

[20] Al-Rashid, S.N.T. (2007) Numerical Simulations of Particle in a Double Oscillators. Journal of University of Anbar for Pure Science, 1.

[21] Charlton, F. (1998) Integrating Factor for First-Order Differential Equations. Classroom Notes, Aston University.

[22] Bessis, N. and Bessis, G. (1997) Open Perturbation and Riccati Equation: Algebraic Determination of Quartic Anharmonic Oscillator Energies and Eigenfunction. Journal of Mathematical Physics, 38, 5483-5492. https://doi.org/10.1063/1.532147

[23] Rogers, G.W. (1985) Riccati Equation and Perturbation Expansion in Quantum Mechanics. Journal of Mathematical Physics, 26, 567-575. https://doi.org/10.1063/1.526592

[24] Nottale, L. and Hermann, R.P. (2003-2017) Private Correspondence. 
Appendix A

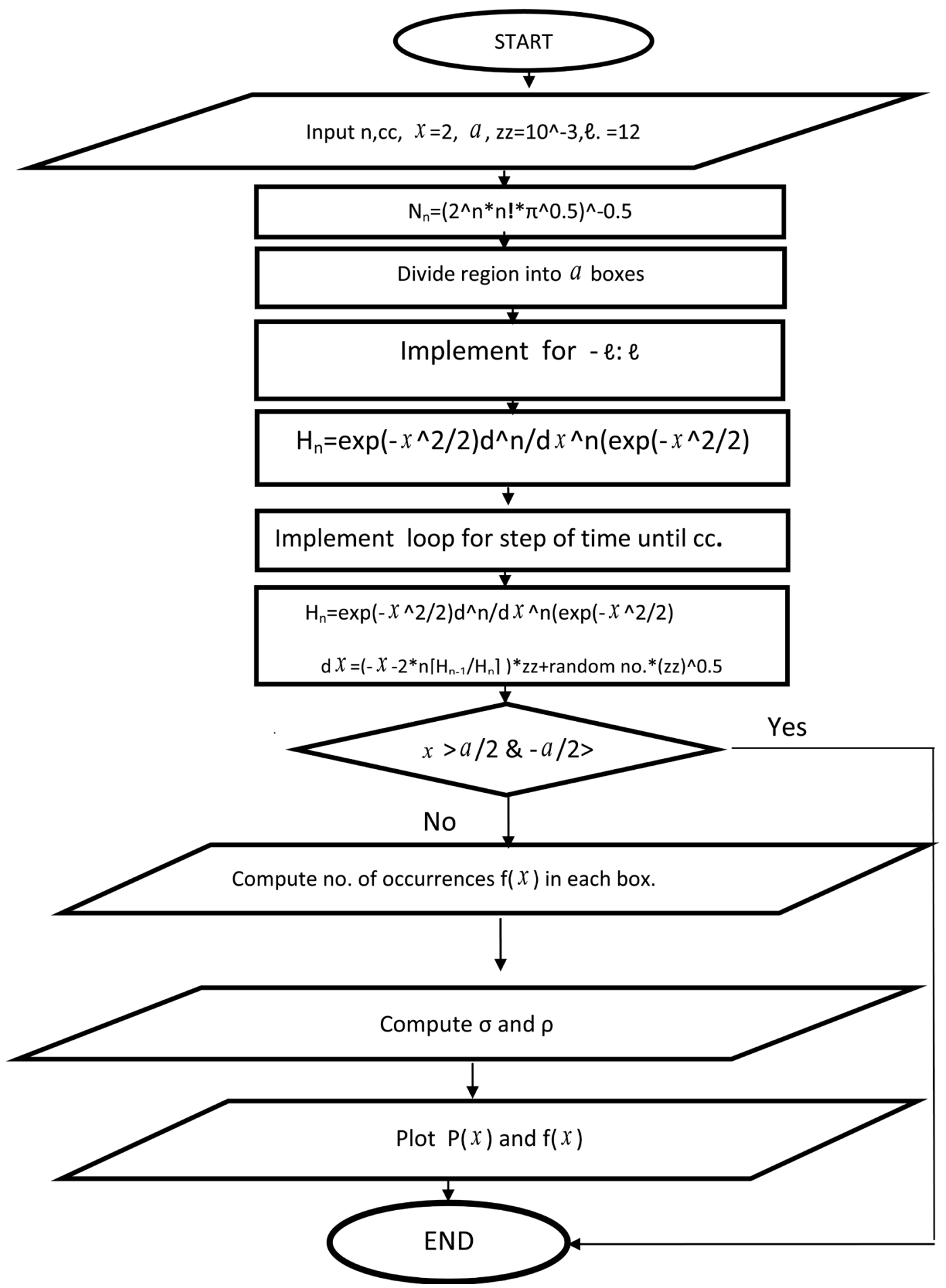

Chart 1. A schematic illustration of the different part of the. Program to calculate probability density of particle in SHO potential. 
Submit or recommend next manuscript to SCIRP and we will provide best service for you:

Accepting pre-submission inquiries through Email, Facebook, LinkedIn, Twitter, etc. A wide selection of journals (inclusive of 9 subjects, more than 200 journals)

Providing 24-hour high-quality service

User-friendly online submission system

Fair and swift peer-review system

Efficient typesetting and proofreading procedure

Display of the result of downloads and visits, as well as the number of cited articles Maximum dissemination of your research work

Submit your manuscript at: http://papersubmission.scirp.org/

Or contact jqis@scirp.org 